\title{
Modelling of the Spatial Evolution of Extreme Laboratory Wave Crest and Trough Heights with the NLS-Type Equations
}

\author{
H.D. Zhang ${ }^{1}$, C. Guedes Soares ${ }^{1 *}$, M. Onorato ${ }^{2}$ \\ ${ }^{1}$ Centre for Marine Technology and Ocean Engineering (CENTEC), Instituto Superior \\ Técnico, Universidade de Lisboa, Portugal \\ ${ }^{2}$ Dipartimento di Fisica, Universitá di Torino, Via P. Giuria 1, 10125 Torino, Italy \\ *Corresponding author e-mail: c.guedes.soares@centec.tecnico.ulisboa.pt
}

\begin{abstract}
The statistical properties of long-crested nonlinear wave time series measured in an offshore basin have been analysed in different aspects such as the distributions of surface elevation, wave crest, wave trough, and wave period. Comparison with linear, second-order and thirdorder theoretical models indicates that although bound wave effects also contribute to the deviation from a Gaussian process, it is the modulational instability that primarily determines the discrepancy in the evolution process in the presence of strong nonlinearity. Interestingly enough, wave crest is more sensitive to the quasi-resonant four-wave interaction effect than wave trough and the scaled maximal wave crest presents a linear regression model with the coefficient of kurtosis. Meanwhile, the estimation of the observed statistical properties is reconstructed on the basis of an ensemble of 100 wave series simulated by the NLS-type equations and compared favourably with the experimental results in most cases. Moreover,
\end{abstract}


with the increased third-order nonlinear effect the difference between NLS and Dysthe simulations is enlarged and mainly reflected on the distribution of wave crest.

Keywords: Bound wave effects; Modulational instability; Third-order nonlinearity; Wave crest; Wave trough; Wave period

\section{Introduction}

In linear random sea state, the relatively large waves are regular in the sense that they do not display any secondary maxima or minima in a wave period [1]. Thus the expected shape of large surface displacements and the distributions of crest and trough can be described reasonably well by Gaussian statistics and Rayleigh model, respectively [2]-[4].

However, the ocean waves are nonlinear, representing by higher, more peaked crests and shallower, more rounded troughs. Consequently, the distribution of surface displacements tends to deviate from the Gaussian form with a positive skewness. Thus an accurate prediction of these features has theoretical importance and practical relevance in ocean engineering such as the prediction of wave forces and structural responses. In deep water, the solution to second order in wave steepness was given by Longuet-Higgins [5], who approximated the distribution of surface elevations in a Gram-Charlier type series, type A in the form of Edgeworth. Tayfun [6] also explored a Stokes-like model and proposed a simple theoretical expression to describe the distribution of nonlinear crests in narrow-band waves. Since then numerous nonlinear crest-height models have been proposed with the similar methods [7]-[11]. For example, some models use nonlinear transforms of Gaussian variables analogous to Gram-Charlier type expansions or fit observational data to a theoretical 
distribution defined in terms of parameters which includes second-order nonlinear effect; some just represent extensions of the narrowband approximations to shallow-water waves or make some heuristic modifications of the Tayfun model; and others require intricate analysis and numerical computations [12]. Most of them can provide valuable insight and work well with various degrees of success in describing the distribution of crests of large waves.

In the past decade, relatively rare and unusually large oceanic waves commonly referred to as abnormal, rogue or freak waves have been intensively researched due to their catastrophic damage to ships and humans. The frequency of occurrence of such waves significantly exceeds the theoretical predictions based on all second-order models, especially in the case of long-crested, steep and narrow-banded waves [13][14]. As a matter of fact, to third order in wave steepness $\varepsilon$, there is a substantial change in the description of water waves. While the bound modes are still present, resonances (or quasi-resonances) are also possible among free waves which consequently can cause a local exponential growth in wave amplitudes within a time scale of the order of $1 / \varepsilon^{2}$ wave periods [15]. The mechanism involved is basically a generalization of the Benjamin-Feir instability [16] or modulational instability [17], which formally applies only to a Stokes wave and a small perturbation.

The numerical simulations of the nonlinear Schrödinger equation have shown that for unidirectional waves, the generation of extreme waves (the tail of the probability density function) depends on the ratio of the wave steepness to the spectral bandwidth [18], which has been named as Benjamin-Feir index, i.e. BFI by Janssen [15]. Comparison with the experimental results of Marintek has proved that the nonlinear Schrödinger equation could catch the modulational instability reasonably well in most cases although an overestimation is 
detected in the sea state with larger initial BFI [19]. A modification of the NLS equation was derived by Dysthe [20] on the basis of a systematic asymptotic procedure. The Dysthe equation or MNLS equation takes into account the fourth order in wave steepness and bandwidth, being capable of representing the asymmetric features of propagating wave packets with narrow spectral bandwidth in deep water [21]-[24]. Further modifications of NLS-type equations have continued such as the spatial form proposed by Lo and Mei [25] for the purpose of performing a convenient comparison with experimental observations, and the one called BMNLS equation appropriate for wider wave spectra, given by Trulsen and Dysthe [26] and Trulsen et al. [27] by adding the higher-order dispersive terms. Actually the spatial variation of coefficient of kurtosis, a potential indication for the formation of extreme waves [28][29], could be reproduced by the numerical simulation of NLS-type models [30] and is almost consistent with the change of rogue wave density [19]. Mori and Janssen [31] contended that the distribution of extreme wave heights can be described by a modified form of the Gram-Charlier series dependent solely on the kurtosis of surface elevations, and further derived a third-order nonlinear model referred to as the modified Edgeworth-Rayleigh distribution under the assumption of narrow-band waves. Later, a more general distribution that can convert to the MER model at some specified moment was given in the work of Tayfun and Fedele [13], where the appropriate forms of the distributions of wave crest and trough, which can include both second-order and third-order nonlinear effects, are proposed as well.

In nature, the ocean waves are not unidirectional and the directional spreading significantly decreases the number of abnormal waves [32]. Consequently the departure from second-order 
theory becomes less accentuated as observed in the three-dimensional wave experiments [33] and confirmed in the numerical simulations with a modified Dysthe equation [34]-[35]. Moreover, further detailed investigation on short-crested waves with different degrees of directionality has been given by Toffoli et al. [36], comparing observed statistics with various numerical simulations.

However, in this paper, concentration is still focused on the unidirectional waves, not only because of the scientific aspect of further understanding the phenomenon, but also due to the conservative situation that abnormal waves occur more frequently in unidirectional than in directional seas [32] and thus this situation is more critical in the design and safe operation of ships and offshore structures [37]. As the observation of extreme waves is really rare in the nature [38], model tests represent an important source for their statistical properties. Hence there is a substantial need for some reliable and computationally efficient numerical tools. The present goal is to analyze the statistical properties of nonlinear wave series recorded from the wave basin of Marintek in different random sea states. At the same time, an estimation of the observed statistical properties is reconstructed by using two different approaches: the NLS equation and the Dysthe equation (MNLS).

This paper is organized as follows: Section 2 gives a short review on the basic theory and numerical models used in this paper. Section 3 briefly describes the laboratory facilities of Marintek and the associated experimental details. Section 4 is devoted to the analysis of various statistical properties and the corresponding comparison with numerical simulations. Section 5 summarizes some important conclusions. 


\section{Theory}

\subsection{Statistical models}

In linear wave theory, the propagation of irregular waves can be represented by the sum of a large number of harmonic and statistically independent wave components, thus according to the central limit theorem, as the number of harmonics increases infinitely the wave surface displacement at one point will tend to Gaussian distribution:

$$
f(\eta)=\frac{1}{\sqrt{2 \pi} \sigma} \exp \left[-\frac{\eta^{2}}{2 \sigma^{2}}\right]
$$

where $\sigma$ is the standard deviation of surface elevation $\eta$. If the wave spectrum is strictly narrow-banded, the envelopes of surface elevations will vary such that the maxima and minima of the upper and lower envelopes coincide exactly with crests and troughs. Under this circumstance, the crest and trough will present the same Rayleigh exceedance distribution [39]:

$$
E_{R}(z)=\exp \left(-\frac{1}{2} z^{2}\right)
$$

where the crest or trough has been scaled with $\sigma$.

In nonlinear waves, the second-order harmonics introduce a vertical asymmetry to the surface profile, rendering wave crests sharper and higher, and troughs shallower and more rounded. Hence, the probability density function of the surface elevation has to be further modified [6]. The approximated form of Tayfun second-order distribution was given by Socquet-Juglard et al. [34]: 


$$
f(\eta)=\frac{1-7 \sigma^{2} k_{p}^{2} / 8}{\sqrt{2 \sigma^{2} \pi\left(1+3 G+2 G^{2}\right)}} \exp \left(-\frac{G^{2}}{2 \sigma^{2} k_{p}^{2}}\right)
$$

where

$$
G=\sqrt{1+2 k_{p} \eta}-1
$$

and $k_{p}$ corresponds to the peak frequency by the linear deepwater dispersion relationship.

Meanwhile, the exceedance distributions of crest $\xi^{+}$and trough $\xi$ amplitudes both deviate noticeably from the Rayleigh form of Eq.(2) and approach the following asymptotic distributions, valid for relatively large waves in the most general case [12][13].

$$
\begin{gathered}
E_{\xi^{+}}(z)=\exp \left[-\frac{1}{2 \mu^{2}}(-1+\sqrt{1+2 \mu z})^{2}\right], \\
E_{\xi^{-}}(z)=\exp \left[-\frac{1}{2} z^{2}\left(1+\frac{1}{2} \mu z\right)^{2}\right],
\end{gathered}
$$

where $\mu$ represents a dimensionless measure of wave steepness and can be defined in a variety of slightly different forms [40]. For unidirectional waves, in the presence of thirdorder nonlinearities, the preceding expressions are further modified as

$$
\begin{gathered}
E_{\xi^{+}}(z)=\exp \left[-\frac{1}{2 \mu^{2}}(-1+\sqrt{1+2 \mu z})^{2}\right]\left[1+\frac{\Lambda}{64} z^{2}\left(z^{2}-4\right)\right], \\
E_{\xi^{-}}(z)=\exp \left[-\frac{1}{2} z^{2}\left(1+\frac{1}{2} \mu z\right)^{2}\right]\left[1+\frac{\Lambda}{64} z^{2}\left(z^{2}-4\right)\right],
\end{gathered}
$$

where

$$
\Lambda=\lambda_{40}+2 \lambda_{22}+\lambda_{04}
$$

and the corresponding fourth-order normalized joint cumulants are defined as

$$
\lambda_{m n}=\left\langle\eta^{m} \hat{\eta}^{n}\right\rangle / \sigma^{m+n}+(-1)^{m / 2}(m-1)(n-1),
$$


where $\hat{\eta}$ represents the Hilbert transform of $\eta$.

On most occasions, the explicit forms of the fourth-order cumulants are not known. However, various approximations are possible to the so-called quasi-Gaussian and longcrested narrow-band waves, although they tend to lack generality. For unidirectional waves with JONSWAP type spectra, normally $\lambda_{40}<3 \lambda_{22}<\lambda_{04}$ [41]. Only in the limit as the spectral bandwidth $v \rightarrow 0, \lambda_{40} \rightarrow 3 \lambda_{22} \rightarrow \lambda_{04}$. Hence these in turn simplify Eq.(9) to

$$
\Lambda_{\text {app }}=8 \lambda_{40} / 3
$$

\subsection{Numerical models}

Starting from the inviscid equations for potential flow, normalized by the characteristic wave number $k_{0}$ and frequency $\omega_{0}$, and assuming constant depth $h$ that is great in comparison with the wave length, i.e. $k_{0} h \square 1$, an assumption is made that the scaled velocity potential $\phi$ and surface displacement $\eta_{0}$ of the wave field can be expanded in harmonic expansions:

$$
\begin{gathered}
\phi=\bar{\phi}+\frac{1}{2}\left(A \mathrm{e}^{i \theta+z_{0}}+A_{2} \mathrm{e}^{2\left(\mathrm{i} \theta+z_{0}\right)}+A_{3} \mathrm{e}^{3\left(\mathrm{i} \theta+z_{0}\right)}+\cdots+\text { c.c }\right), \\
\eta_{0}=\bar{\eta}+\frac{1}{2}\left(B \mathrm{e}^{i \theta}+B_{2} \mathrm{e}^{2 \mathrm{i} \theta}+B_{3} \mathrm{e}^{3 \mathrm{i} \theta}+\cdots+\text { c.c }\right),
\end{gathered}
$$

where c.c. denotes the complex conjugate, and the phase is $\theta=x-t$ for a wave with carrier wave vector in the $x$-direction. $\bar{\phi}$ and $\bar{\eta}$ are real functions slowly varying in space and time, representing the mean flow and surface elevation brought about by the radiation stress of the wave, and $A, A_{\mathrm{n}}, B, B_{\mathrm{n}}$ are complex harmonic amplitudes. Trulsen et al. [27] explained how the MNLS equation can be enhanced with the exact linear dispersion by introducing a pseudo-differential operator for the linear part. By expanding the linear pseudo-differential 
operators in power series expansions and truncating at appropriate orders, the MNLS equation of Dysthe [20] and the broader bandwidth equation of Trulsen and Dysthe [26] can be recovered. Furthermore by truncating the nonlinear part to retain only the leading cubic nonlinear term, the standard nonlinear Schrödinger equation is recovered as well. In one horizontal dimension, the operator is algebraic, leading to the following MNLS equation with a moving frame at the wave group velocity:

$$
\begin{gathered}
\frac{\partial B}{\partial x}+i \frac{\partial^{2} B}{\partial t^{2}}+i|B|^{2} B-8|B|^{2} \frac{\partial B}{\partial t}-2 B^{2} \frac{\partial B^{*}}{\partial t}-\left.4 i B \frac{\partial \bar{\phi}}{\partial t}\right|_{z=0}=0 \\
\frac{\partial \bar{\phi}}{\partial z}=-\frac{\partial|B|^{2}}{\partial t}, \quad z=0, \\
\frac{\partial^{2} \bar{\phi}}{\partial z^{2}}+4 \frac{\partial^{2} \bar{\phi}}{\partial t^{2}}=0, \quad-\bar{h}<z<0, \\
\frac{\partial \bar{\phi}}{\partial z}=0, \quad z=-\bar{h} .
\end{gathered}
$$

and the cubic nonlinear Schrödinger (NLS) equation is

$$
\frac{\partial B}{\partial x}+i \frac{\partial^{2} B}{\partial t^{2}}+i|B|^{2} B=0
$$

The reconstruction of the scaled surface displacement in Eq.(13) is achieved by the formulae

$$
\bar{\eta}=-\frac{\partial \bar{\phi}}{\partial t}, B_{2}=\frac{1}{2} B^{2}+\mathrm{i} B \frac{\partial B}{\partial t}, B_{3}=\frac{3}{8} B^{3} .
$$

The present implementation is based on the imposed periodic boundary conditions in time, which is appropriate for the experimental data under consideration. The detailed numerical method has been documented in other papers [25] [34] [42][43]. In a word, the nonlinear part 
is integrated by a finite difference method in physical space, while the linear dispersive part is solved exactly in Fourier space [21].

In order to compare with the laboratory experiments, the initial condition for the numerical simulations is typical of sea states described by the JONSWAP power spectrum.

$$
S(\omega)=\alpha g^{2} \omega^{-5} \exp \left[-\frac{5}{4}\left(\frac{\omega_{p}}{\omega}\right)^{4}\right] \gamma^{\exp \left[-\left(\omega-\omega_{p}\right)^{2} /\left(2 \sigma_{0}^{2} \omega_{p}^{2}\right)\right]}
$$

where $\sigma_{0}=0.07$ if $\omega \leq \omega_{p}$ and $\sigma_{0}=0.09$ if $\omega>\omega_{p}$. Here $\omega_{p}$ is the peak frequency, $\gamma$ is the peak enhancement parameter, and $\alpha$ is the Phillips' constant related with the significant wave height $H_{s}$. As $\gamma$ increases, the spectrum becomes higher and narrower around the peak frequency. In the present simulations, $\gamma=3$, and $\varepsilon=k_{p} H_{s} / 2$ [44]. The initial JONSWAP random surface elevation has been synthesized as sums of independent harmonic components, by means of the inverse Fast Fourier Transform of complex random Fourier amplitudes which are prepared according to the "random realization approach" by using random spectral amplitudes as well as random phases [45].

\section{Facilities and Experimental Data}

The experiments were carried out in the wave basin of Marintek, Trondheim, in 1999 with dimensions $80 \mathrm{~m}$ long and 50m wide as shown in Fig. 1. The wave surface elevations were measured by 10 capacitance wave gauges uniformly deployed at $5 \mathrm{~m}$ distance along the centre line of the basin where the water depth is $2 \mathrm{~m}$. The gauge closest to the wave maker is at $10 \mathrm{~m}$ distance. The double-flap wave-maker is installed at one of the short walls of the basin to generate the unidirectional waves and the sloping beach at the opposite side serves to absorb 
the incident wave energy [19]. What needs to be mentioned here is that this data set has been used in many papers to compare with various theoretical models and no lack of consistency was observed, which could be attributed to this reflection. Moreover, the statistical analysis of the first $20 \mathrm{~min}$ series is almost consistent with the results of $27 \mathrm{~min}$ series, meaning that these signals are free of possible contamination.

The length scale of the experiment is 1:50. The duration of each run of the experiment is around 27 minutes and hence nearly 3 hours at full-scale. All the initial parameters of these experiments are listed in Table 1 where $8241-45$ is a set of records from five tests run under the same model condition. According to the initial wave steepness $\varepsilon$, these four experiments could be categorized into three groups: the low (8201), moderate $(8202,8241)$ and severe (8219) sea states, and will be represented by different symbols in the later analysis. In the following comparison, the measured data mainly come from gauge 1, 5 and 8 which could represent the initial, intermediate and peak stage in wave evolution process respectively.

In the laboratory experiments, the spectra generated at the wave maker is unidirectional and narrow-banded. Each experiment uses different sets of random phases and the variance of amplitudes is such that the spectrum of waves generated at the wave maker represents a JONSWAP spectrum.

With regard to the numerical simulation, the sea states in Table 1 have been realized by NLS and MNLS equations simultaneously with the same initial conditions. For the purpose of eliminating the variability in the following statistical analysis, 100 numerical realizations of the random surface elevation have been performed for each sea state in this paper. 


\section{Comparisons}

\subsection{Statistical Parameters}

As pointed out before, in order to achieve statistically significant results, a large number of time series are needed in the numerical simulations. In this respect, Fig. 2 verifies that the aforementioned number of realizations can lead to stable estimates of the statistical moments. Obviously, if the number of time series is less than five, the estimation of coefficient of kurtosis is normally not reliable. In contrast, the coefficient of skewness is much stable and does not display such a large variation among different wave series although it is not presented here. Moreover, the spatial evolutions of these two parameters in different models are analyzed and compared in detail in our past work [46].

In second-order narrowband wave theory, a quadratic function can be derived between the coefficient of kurtosis and the coefficient of skewness [8], and the similar relation was found by Guedes Soares et al. [28] on the basis of full-scale field data. However, in Fig. 3 the quadratic relation only catches the tendency of variation without giving impressive agreement for the reason that the coefficient of kurtosis is notably influenced by the dynamics of the free modes of four wave interactions while the skewness is only weakly affected. To a certain degree, the stable relationship indicated by the numerical simulations is consistent with the experimental results in Marintek and those presented in the work of Mori et al. [47].

As indicated in Fig. 4, even though the bound wave effects are included, $\Lambda$ is still equal to $\Lambda_{\text {app }}$ in the case of linear wave series, e.g., the sea state 8201 (square) in this paper. Once the nonlinearity increases, $\Lambda$ will become a little smaller than $\Lambda_{\text {app }}$ which is obviously opposite to 
the second-order theoretical conclusion obtained by Tayfun and Fedele [13]. According to the previous research [19] [24], it is found that the modulational instability does not contribute too much to the discrepancy between these two parameters in the absence of bound wave effects. Therefore, it seems that the difference is caused by the combined effects of modulational instability and bound wave effects. Furthermore, in the numerical simulation, the third-order nonlinear model apparently overestimates the fourth-order cumulants in the severe (8219) sea state and NLS equation cannot identify the difference between these two parameters even though considering the second-order bound wave effects. On the other hand, the numerical model of Dysthe equation can provide a better description on their relationship. Now it is clear that the higher-order bound wave effects are enlarged by the modulational instability, which consequently leads to $\Lambda_{\text {app }}>\Lambda$. However, as a common sense, a larger ensemble of nonlinear wave series normally presents a larger fourth-order cumulants, which is obviously not true in the Dysthe simulation. According to the above discussion, the parameter $\Lambda$ will be adopted in the third-order nonlinear model in the prediction of exceedance distribution of wave crests and troughs.

It is well known that a linear regression model exists between the scaled maximum wave height and the coefficient of kurtosis, which is reported by many researchers such as Guedes Soares et al. [28] after analyzing all sea state records during the storm of November 1997 in North Alwin and Draupner's storm in the beginning of 1995. Moreover, as indicated in Fig. 5, the similar linear relationship is also detected for the scaled maximum wave crests rather than the scaled maximum wave trough in both experimental and numerical results. Hence, it could be concluded that the abnormal wave normally generates with a very large wave crest, the 
formation of which is dominated by the nonlinear interaction among free modes.

\subsection{Surface Elevation}

Fig. 6 presents the probability density functions of the surface elevations measured at Gauge 8 where the modulational instability has almost been fully developed in all random sea states. For convenience, the surface elevations are scaled by the standard deviation $\sigma$ of the concurrent time series. The experimental probability density function is also compared with the Gaussian distribution in Eq.(1) and the Tayfun second-order model in Eq.(3).

As the initial wave steepness increases, i.e. Fig. $6 a \sim$ Fig. 6 d, the wave surface elevations begin to deviate from the normal distribution noticeably, becoming narrower and positive skewed which can be captured by the second-order model of Tayfun in most part except for the peak values in the severe (8219) and second moderate (8241) sea states. Therefore, the deviation in this range is mainly attributed to the bound wave effects. Meanwhile, the NLStype simulations are both consistent with the experimental results up to 4 standard deviations in all sea states.

To further explore the nonlinear effect on the formation of larger surface elevation, different axes has been applied on the same distribution, as shown on the right panel of Fig. 6 . It is clear that the positive skew indicates the tail on the right side is longer and fatter than the left side. In agreement with the previous experimental and numerical studies [33][34] [36], if the wave series is strongly nonlinear, a substantial deviation from the Gaussian and second- 
order models could be observed on both tails of the wave surface distribution, in particular in the positive section. Considering the third-order or higher-order bound wave effect is much smaller, the considerable discrepancy in this part is mainly attributed to the modulational instability induced by the nonlinear dynamics of free wave modes [48]. Although the NLStype models are not fully nonlinear, they are both able to capture the aforementioned deviations reasonably well. Moreover, as the sea state becomes more and more severe, the NLS equation tends to overestimate the positive tail of wave surface distribution due to no energy dissipation and unsuppressed stronger nonlinear effect (comparing to Dysthe equation) in numerical simulations. The spatial variation of surface elevation downstream the wave basin is consistent with what observed in the work of Onorato et al. [33] where the directional effect has been taken into account in different sea states as well.

\subsection{Wave Crest}

Fig. 7 and Fig. 8 display the spatial variation of exceedance distribution of wave crests, which are defined as the highest elevation of each individual wave with respect to the mean water level. For the crest amplitude, the second-order interaction should participate in the deviation from Gaussian statistics. Therefore, apart from the linear Rayleigh distribution of Eq.(2) and the third-order nonlinear model of Eq.(7), the second-order distribution in Eq.(5) is also compared with the experimental data in the following discussion. Meanwhile, the numerical results based on NLS-type equations are also plotted.

In the low sea state (8201), at the initial and intermediate stage, i.e. Fig. 7a and Fig. 7b, both second-order and third-order nonlinear models can describe the wave crest distribution 
very well because the nonlinearity is really weak and basically arisen from the second-order bound wave effect. However, at the peak stage, i.e. Fig. 7c, third-order modulation begin to but not fully work on the generation of larger amplitude of crest, indicated by the obvious deviation of observed statistics from second-order nonlinear model. The two numerical simulations are almost consistent with each other and fit the experiments perfectly from the point of view of statistics.

In the first moderate sea state (8202), Fig. 7d Fig. 7f, only the third-order GC model can describe the crest-height statistics reasonably well in the whole evolution process, meaning that the bound wave effect is relatively weak compared with the Benjamin-Feir type modulational instability. Although the two numerical simulations begin to deviate from each other at the larger amplitude part, they both can catch the major statistics of wave crest in a short-term wave series.

In the second moderate sea state (8241), five similar wave series with the same initial wave parameter not only guarantee a stable observation in some statistical variable such as the coefficient of kurtosis [19] [30], but also generate a much larger maximal crest height. Additionally, the smaller wave peak period in this sea state means a much longer relative spatial evolution, up to 36 wave lengths corresponding to the peak of the spectrum at the wave-maker. It is found that the third-order GC model is still the best choice on describing the wave crest distribution. Moreover, as discussed on the maximal wave height distribution [31] [49], the number of waves also plays an important role on the prediction of maximal crest height. Moreover, except for the initial stage, it seems that in this case the numerical 
simulation of NLS is more consistent with these experimental results than those given by Dysthe equation.

In the severe sea state (8219), Fig. 8d Fig. 8f, the third-order GC model still works but some difference is noticeable on the tail of the distribution because in addition to the strong nonlinearity, wave breaking (see Fig. 8e) begins to play an important role on the evolution process as well, which could not be included in the existing potential theory [50]. With regard to the numerical simulation, it seems that the MNLS equation presents an agreement with the experiment in the initial and intermediate stages while the NLS equation in the peak stage. However, as we know the difference of MNLS and NLS should come from the spectral width and neither of them fails in the local strong nonlinearity. It would be expected, although not given here, that the spectrum is broadest at the peak stage and therefore NLS would not be a better approximation than MNLS. Hence the controversy here may be attributed to the uncertainty and variation in the rough laboratory experiments. Agreeing on the limited statistical estimate alone cannot be a very sound evidence for the validity of NLS at the peak stage.

\subsection{Wave Trough}

The same comparison as those in wave crest distribution has been applied in the wave trough as well. In the low sea state (8201), i.e. Fig. 9a Fig. 9c, the spatial variation of trough height is more or less the same as that observed in the crest height. The numerical results governed by NLS-type equations both tend to Rayleigh distributed, comparing favourably with the laboratory observations in most cases. 
In the first moderate sea state (8202), i.e. Fig. 9d Fig. 9f, second-order and third-order nonlinear models do not exhibit too much difference in fitting the experimental results at the initial and intermediate stages. Only in the peak stage, the effect of modulational instability has been fully developed, causing the observed statistics to deviate from the conventional second-order model of NB noticeably [51]. Compared with those deviations in wave crest under the same sea state, it could be concluded that wave trough is less sensitive than wave crest to modulational instability. It is also amazing that the two numerical simulations are still in agreement with each other and fit the observe statistics reasonably well.

In the second moderate sea state (8241), the effect of modulational instability is only noticeable in Fig. 10b and Fig. 10c, meaning that wave trough reaches the fully developed condition much later than the wave crest. Considering that the coefficient of kurtosis also stops growing at Gauge 5, corresponding to the location of Fig. 10b (see [19]), it can be argued that the third-order nonlinearity contributes mainly to the wave crest, and that after crest and trough are both fully developed, the direct indication is the coefficient of kurtosis with an approximately stable value.

Moreover, as displayed in Figs. 10b, 10c and 10f [52][53], the wave trough normally can be fitted surprisingly well by the linear Rayleigh model if the wave series have been fully worked by the third-order four-wave interactions. As expected, wave breaking also has an influence on the wave trough distribution, as illustrated on the tail of Fig. 10e which is in agreement with the observed statistics of crest in Fig. 8e. Although the NLS-type equations can not include the effect of wave breaking, they have caught the observed statistics of troughs very well and kept in conformity with each other even in the presence of stronger 
nonlinearity. Consequently, it can be said that the major discrepancy of wave height distribution between numerical simulations principally arises from the difference in the wave crest distribution. In other words, the wave crest is more sensitive than wave trough to the third-order quasi-resonant four-wave interaction that takes place near the peak of the spectrum.

\subsection{Wave Period}

Comparison of observed wave period distribution with the theoretical model of LonguetHiggins [54] has been presented in the earlier work [55]. In this section, it is only focused on the numerical and experimental results measured in Gauge 8 for the reason that the spatial variation of wave period is not very distinct except for being a little narrower detected downstream the wave tank. In Fig. 11a Fig. 11d, it is evident that the periods of individual waves in a wave train exhibit a distribution narrower than that of wave crests or troughs, and the spread lies mainly in the range of 0.5 to 1.5 times the mean wave period which is a little narrower than the scope of ocean waves proposed by Goda [56]. Moreover, as the sea states become more and more severe (from top to bottom), the observed wave period distribution will become much steeper and narrower as well. It is also noticeable that the observed statistics generates a hump in the interval $(0.5,1)$ in the low sea state (see Fig. 11a) and the same phenomena are also observed in the laboratory experiments carried out in the offshore basin of CEHIPAR, Spain [55]. The NLS-type numerical simulations could catch the fundamental properties of wave period distribution in all cases, in particular in the first moderate sea state (8202), i.e. Fig. 11b. It is also surprising that the tails on the left and right sides of wave period distribution are fitted quite well by both numerical simulations, as 
presented on the right column of Fig. 11. The little variation of the tail among different sea states indicates that the modulational instability enlarges the magnitude of wave crest without apparently increasing the associated wave period. Finally, what needs to be pointed out is that the spatial variation of wave period in numerical simulations could be neglected as well.

\section{Conclusions}

In this paper, some statistical properties of nonlinear time wave series in different sea states has been analyzed and compared with the existing linear and nonlinear theoretical predictions. Meanwhile, their estimations of numerical simulations governed by NLS-type equations, i.e., NLS and Dysthe equations, are also presented on an ensemble of 100 similar wave series.

It is confirmed again that some relationships derived in second-order wave theory are not valid any more in the presence of modulational instability. For example, the quadratic function between coefficients of skewness and kurtosis tends to underestimate their variation. $\Lambda$ becomes a little smaller than $\Lambda_{\text {app }}$ as a result of the combined effects of modulational instability and bound wave effects. Furthermore, a linear regression model exists between the scaled maximum wave crest and the coefficient of kurtosis, meaning that the abnormal wave normally appears with a very large wave crest.

With the increased nonlinearity, the wave surface elevations begin to deviate from the Gaussian process, becoming narrower and positively skewed in the domain close to the mode of distribution. Comparisons with various theoretical models indicates that the deviation in this range except for the peak value is mainly attributed to the bound wave effects while for 
the larger amplitude of surface elevation, especially in the positive part, the difference is principally caused by the modulational instability. Moreover, the NLS-type simulations are both consistent with the experimental results up to 4 standard deviations in all sea states. Furthermore, its spatial variation downstream the wave basin is in agreement with that observed in the work of Onorato et al. [33] where the directional effect has been taken into account as well.

As for the crest amplitude, it is well known that the second-order interaction participates in the deviation from Gaussian statistics. If the nonlinearity is weak, the wave crest distribution could be fitted well by both second-order and third-order nonlinear models due to the limited nonlinear effects. Once the nonlinearity increases, e.g. in the moderate sea state, the modulational instability will immediately dominate the nonlinearity effect and generate larger amplitude of crest which could only be described by the third-order GC model. Similarly as observed in wave height distribution, the maximum crest is also determined by the number of waves in the considered series.

In the same sea state, a similar spatial variation of wave trough is detected as well, but it is not as sensitive as wave crest to modulational instability. The quasi-resonant four-wave interaction largely dominates the evolution of wave profile, generating a sharper and higher crest easily and also creating a deeper trough when the nonlinearity is fully developed which can be indicated by an approximately stable value of coefficient of kurtosis. It is also amazing that the two numerical simulations are still in agreement with each other and fit the observed statistics reasonably well in most cases. Obviously, the major discrepancy between numerical 
simulations exhibited in the wave height distribution is primarily attributed to the difference in wave crest distribution.

The spatial variation of wave period is not very obvious except for becoming a little narrower along the wave tank. Moreover, the period distributions in a wave train are much narrower than that of wave crests or troughs, and the spread lies mainly in the range of 0.5 to 1.5 times the mean wave period. With the increased nonlinearity in these four sea states, the observed wave period distribution becomes steeper and narrower, while this tendency could not be identified by the numerical simulations. However, the main characteristics are still reflected correctly by NLS-type equations. The little variation on the right tail of wave period distribution reveals that the modulational instability does not enlarge the wave period noticeably.

\section{Acknowledgements}

The first author gratefully acknowledges O. Gramstad, L. Shemer and A. Slunyaev for interesting discussions and their help in solving numerical problems related to the development of the present code of Dysthe formulation. Financial support by Portuguese Foundation for Science and Technology under the grant SFRH/BD/98983/2013 is also acknowledged. M.O. was supported by ONR Grant No. 214N000141010991 and by MIUR Grant PRIN 2012BFNWZ2. M.O. thanks Dr. B. Giulinico for fruitful discussions.

\section{References}

[1] Longuet-Higgins MS. The statistical analysis of a random moving surface. Philosophical Transactions of the Royal Society London A 1957; 966: 321-387. 
[2] Lindgren G. Some properties of a normal process near a local maximum. Annals of Mathematical Statistics 1970; 4(6): 1870-1883.

[3] Lindgren G. Local maxima of Gaussian fields. Arkiv för Matematik 1972; 10: 195-218.

[4] Boccotti P. Wave Mechanics for Ocean Engineering. Elsevier Science 2000.

[5] Longuet-Higgins MS. The effects of non-linearities on statistical distributions in the theory of sea waves. Journal of Fluid Mechanics 1963; 17: 459-480.

[6] Tayfun MA. Narrow-band nonlinear sea waves. Journal of Geophysical Research 1980; 85 (C3): 1548-1552.

[7] Winterstein SR. Nonlinear vibration models for extremes and fatigue. Journal of Engineering Mechanics 1988; 114: 1772-1790.

[8] Marthinsen T. Winterstein SR. On the skewness of random surface waves. In: Proceedings of $2^{\text {nd }}$ International Society of Offshore and Polar Engineering, ISOPE, San Francisco, 1992; 3:472-478.

[9] Kriebel DL. Dawson TH. Nonlinearity in crest height statistics. In: Proceedings of $2^{\text {nd }}$ International Conference Wave Measurement \& Analysis, ASCE, New Orleans, 1993; $61-75$

[10] Forristall GZ. Wave crest distributions: observations and second-order theory. Journal of Physical Oceanography 2000; 30 (8): 1931-1943. 
[11] Arena F. Fedele F. A family of narrow-band nonlinear stochastic processes for the mechanics of sea waves. European Journal of Mechanics B/Fluids 2002; 21: 125-137.

[12] Fedele F. Tayfun MA. On nonlinear wave groups and crest statistics. Journal of Fluid Mechanics 2009; 620: 221-239.

[13] Tayfun MA. Fedele F. Wave-height distributions and nonlinear effects. Ocean Engineering 2007; 34: 1631-1649.

[14] Cherneva Z. Tayfun MA. Guedes Soares C. Statistics of nonlinear waves generated in an offshore wave basin. Journal of Geophysical Research 2009; 114 (C08005), doi: 10.1029/2009JC005332.

[15] Janssen PAEM. Nonlinear four-wave interactions and freak waves. Journal of Physical Oceanography 2003; 33: 863-884.

[16] Benjamin TB. Feir JE. The disintegration of wave trains on deep water. Part 1. Theory, Journal of Fluid Mechanics 1967; 27: 417-430.

[17] Zakharov VE. Stability of periodic waves of finite amplitude on a surface of deep fluid. Journal of Applied Mechanics and Technical Physics 1968; 9: 190-194.

[18] Onorato M. Osborne A. Serio M. Bertone S. Freak waves in random oceanic sea states. Physical Review Letters 2001; 86, doi: 10.1103/PhysRevLett.86.5831.

[19] Zhang HD. Cherneva Z. Guedes Soares C. Onorato M. Comparison of distributions of wave heights from nonlinear Schrödinger equation simulations and laboratory 
experiments. Proceedings of the 32nd International Conference on Ocean, Offshore and Arctic Engineering, Nantes, France, OMAE 2013-11633, 2013.

[20] Dysthe KB. Note on a modification to the nonlinear Schrödinger equation for application to deep water waves. Proceedings of the Royal Society of London A 1979; 369: $105-114$.

[21] Trulsen K. Stansberg CT. Spatial evolution of water surface waves: numerical simulation and experiment of bichromatic waves. In: Proceedings of the Eleventh (2001) International Offshore and Polar Engineering Conference, Stavanger, Norway, 2001; 7177.

[22] Shemer L. Kit E. Jiao H. Eitan O. Experiments on Nonlinear Wave Groups in Intermediate Water Depth. Journal of Waterway, Port, Coastal, and Ocean Engineering, 1998; 124: 320-327.

[23] Shemer L. Sergeeva A. Slunyaev A. Applicability of envelope model equations for simulation of narrow-spectrum unidirectional random wave field evolution: Experimental validation. Physics of Fluids 2010; 22 (016601).

[24] Zhang HD. Guedes Soares G. Cherneva Z. Onorato M. Modeling extreme wave heights from laboratory experiments with the nonlinear Schrödinger equation. Natural Hazards and Earth System Sciences; 14: 959-968.

[25] Lo E. Mei CC. A numerical study of water-wave modulation based on a higher-order nonlinear Schrödinger equation. Journal of Fluid Mechanics 1985; 150: 395-416. 
[26] Trulsen K. Dysthe KB. A modified nonlinear Schrödinger equation for broader bandwidth gravity waves on deep water. Wave Motion 1996; 24: 281-289.

[27] Trulsen K. Kliakhandler I. Dysthe KB. Velarde MG. On weakly nonlinear modulation of waves on deep water. Physics of Fluids 2000; 12: 2432-2437.

[28] Guedes Soares C. Cherneva Z. Antão E. Characteristics of abnormal waves in North Sea storm sea states. Applied Ocean Research 2003; 25: 337-344.

[29] Guedes Soares C. Cherneva Z. Antão E. Abnormal waves during the hurricane Camille. Journal of Geophysical Research 2004; 109 (C08008).

[30] Onorato M. Osborne A. Serio M. Cavaleri L. Modulational instability and non-Gaussian statistics in experimental random water-wave trains. Physics of Fluids 2005; 17 (078101).

[31] Mori N. Janssen P. On kurtosis and occurrence probability of freak waves. Journal of Physical Oceanography 2006; 36: 1471-1483.

[32] Onorato M. Osborne A. Serio M. Extreme wave events in directional random oceanic sea states. Physics of Fluids 2002; 14 (4): 25-28.

[33] Onorato M. Cavaleri L. Fouques S. Gramstad O. Janssen PAEM. Monbaliu J. Osborne AR. Pakozdi C. Serio M. Stansberg CT. Toffoli A. and Trulsen K. Statistical properties of mechanically generated surface gravity waves: a laboratory experiment in a threedimensional wave basin. Journal of Fluid Mechanics 2009; 627: 235-257.

[34] Socquet-Juglard H. Dysthe K. Trulsen K. Krogstad HE. Liu J. Distribution of surface gravity waves during spectral changes. Journal of Fluid Mechanics 2005; 542: 195-216. 
[35] Gramstad O. Trulsen K. Influence of crest and group length on the occurrence of freak waves. Journal of Fluid Mechanics 2007; 582: 463-472.

[36] Toffoli A. Gramstad O. Trulsen K. Monbaliu J. Bitner-Gregersen EM. Onorato M. Evolution of weakly nonlinear random directional waves: laboratory experiments and numerical simulations. Journal of Fluid Mechanics 2010; 664: 313-336.

[37] Guedes Soares C. Fonseca N. Pascoal R. Abnormal wave induced load effects in ship structures. Journal of Ship Research 2008; 52 (1): 30-44.

[38] Guedes Soares C. Cherneva Z. Petrova PG. Antao E. Large Waves in Sea States. In: Marine Technology and Engineering. Guedes Soares C. Garbatov Y. Fonseca N. Teixeira AP. (Eds.), Taylor \& Francis Group, London, 2011; 79-95.

[39] Longuet-Higgins MS. On the statistical distribution of heights of sea waves. Journal of Marine Research 1952; 11: 245-266.

[40] Tayfun MA. Statistics of nonlinear wave crests and groups. Ocean Engineering 2006; 33: $1589-1622$.

[41] Tayfun MA. Lo J. Nonlinear effects on wave envelope and phase. Journal of Waterway, Port, Coastal, and Ocean Engineering 1990; 116: 79-100.

[42] Trulsen K. Dysthe KB. Frequency downshift in three-dimensional wave trains in a deep basin. Journal of Fluid Mechanics 1997; 352: 359-373.

[43] Slunyaev A. Pelinovsky E. Guedes Soares C. Modeling Freak Waves from the North Sea. Applied Ocean Research 2005; 27: 12-22. 
[44] Onorato M. Osborne A. Serio M. Cavaleri L. Brandini C. Stansberg C. Extreme waves, modulational instability and second order theory: wave flume experiments on irregular waves. European Journal of Mechanics B/ Fluids 2006; 25: 586-601.

[45] Onorato M. Proment D. Nonlinear interactions and extreme waves: Envelope equations and experimental results. In: Marine Technology and Engineering. Guedes Soares C. Garbatov Y. Fonseca N. Teixeira AP. (Eds.), Taylor \& Francis Group, London, 2011; $135-146$.

[46] Zhang HD. Guedes Soares G. Onorato M. Modelling of the spatial evolution of extreme laboratory wave heights with the nonlinear Schrödinger and Dysthe equations. Ocean Engineering 2014; 89: 1-9.

[47] Mori N. Onorato M. Janssen PA. Osborne AR. Serio M. On the extreme statistics of long-crested deep water waves: Theory and experiments. Journal of Geophysical Research 2007; 112 (C09011), doi:10.1029/2006JC004024.

[48] Fedele F. Cherneva Z. Tayfun MA. Guedes Soares C. Nonlinear Schrödinger invariants and wave statistics. Physics of Fluids 2010; 22: 036601-1-036601-9, http: // dx.doi.org $/ 10.1063 / 1.3325585$.

[49] Mori N. Onorato M. Janssen PA. On the estimation of the kurtosis in directional sea states for freak wave forecasting. Journal of Physical Oceanography 2011; 41: 1484-1497.

[50] Bitner-Gregersen EM. Toffoli A. On the probability of occurrence of rogue waves. Natural Hazards and Earth System Sciences 2012; 12: 751-762. 
[51] Toffoli A. Bitner-Gregersen EM. Onorato M. Babanin AV. Wave crest and trough distributions in a broad-banded directional wave field. Ocean Engineering 2008; 35: $1784-1792$.

[52] Toffoli A. Onorato M. Bitner-Gregersen EM. Osborne AR. Babanin AV. Surface gravity waves from direct numerical simulations of the Euler equations: A comparison with second-order theory. Ocean Engineering 2008; 35: 367-379.

[53] Shemer L. Sergeeva A. An experimental study of spatial evolution of statistical parameters in a unidirectional narrow-banded random wave field. Journal of Geophysical Research 2009; 114 (C01015), doi: 10.1029/ 2008JC005077.

[54] Longuet-Higgins MS. On the joint distribution of wave periods and amplitudes in a random wave field. Proceeding of the Royal Society London A 1983; 389: 241-258.

[55] Zhang HD. Cherneva Z. Guedes Soares C. Joint distributions of wave height and period in laboratory generated nonlinear sea states. Ocean Engineering 2013; 74: 72-80.

[56] Goda Y. Random seas and design of maritime structures. Advanced Series on Ocean Engineering 15, World Scientific, 2000. 
Table 1. Parameters of the four different experiments.

\begin{tabular}{cccccc}
\hline No. & $H_{s}(\mathrm{~m})$ & $T_{p}(\mathrm{~s})$ & $\varepsilon$ & $\gamma$ & Symbol \\
\hline 8201 & 3.5 & 10 & 0.070 & 3.0 & Square \\
8202 & 7.0 & 10 & 0.141 & 3.0 & Diamond \\
$8241-45$ & 3.5 & 7 & 0.144 & 3.0 & Circle \\
8219 & 9.0 & 10 & 0.181 & 3.0 & Triangle
\end{tabular}

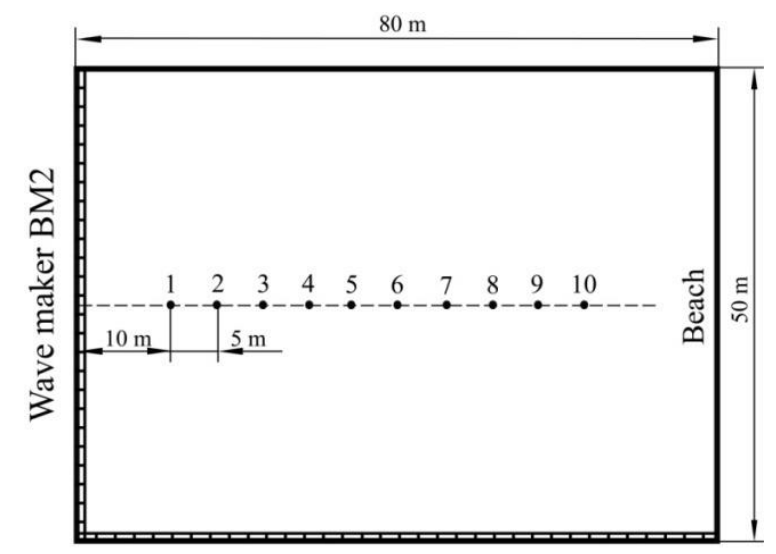

Fig. 1. Layout of the Marintek wave basin.

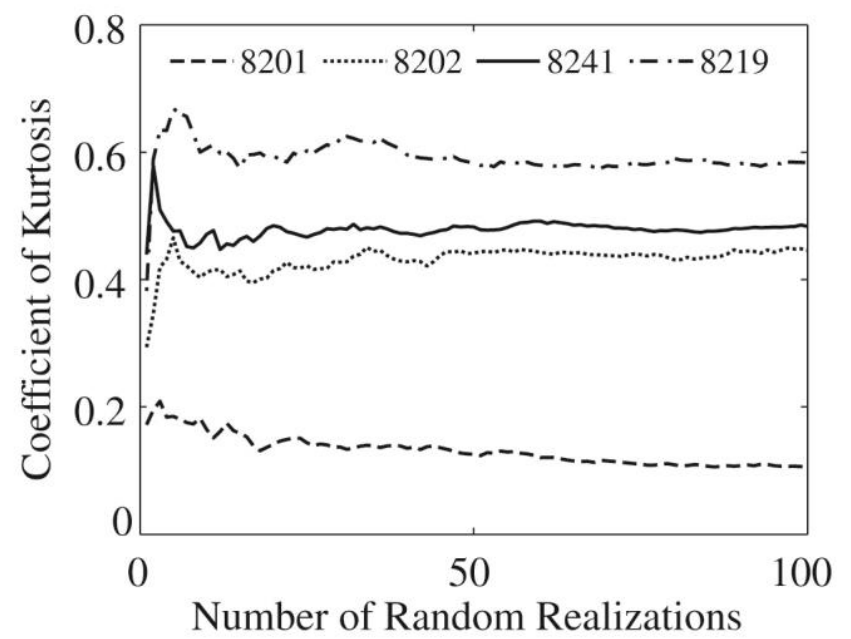

Fig. 2. Coefficient of kurtosis as a function of the number of random realizations in Dysthe simulation. 


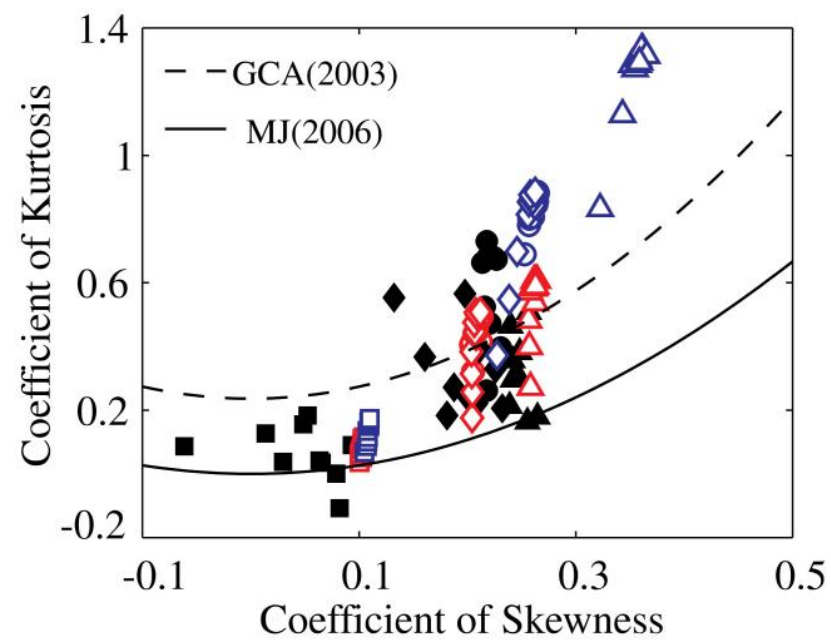

Fig. 3. Coefficient of kurtosis as a function of coefficient of skewness. The dashed line is the empirical fit by Guedes Soares et al. [28] while the solid line is proposed by Mori and Janssen [31]. The black solid symbols are experimental data, and the blue and red empty symbols are simulated data derived by NLS and MNLS equation respectively. Different symbols represent different sea states as explained in Table 1.

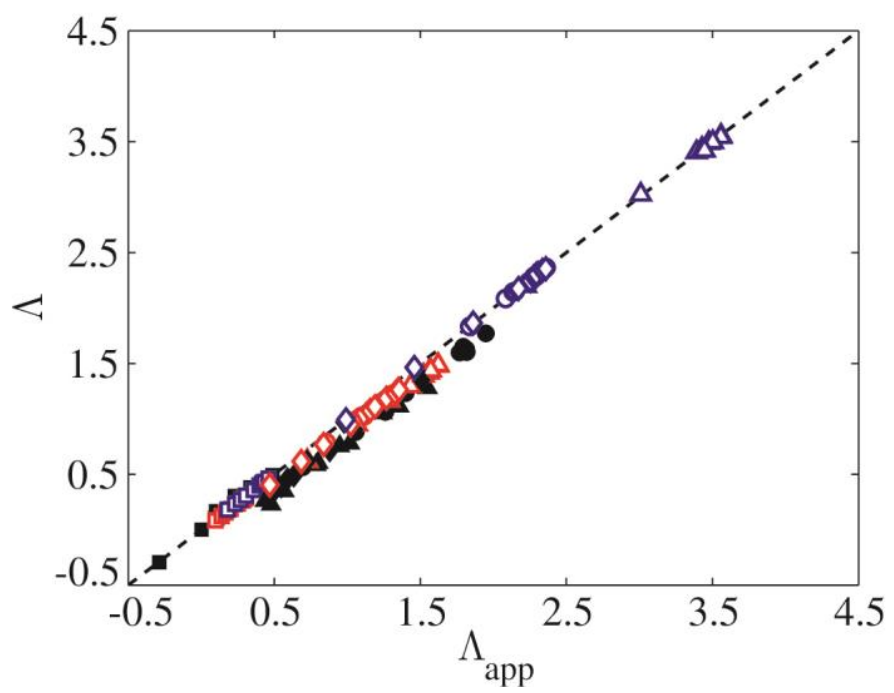

Fig. 4. Relationship between $\Lambda$ and $\Lambda_{\text {app. }}$. The meanings of marks are the same as those in Fig. 3 . 


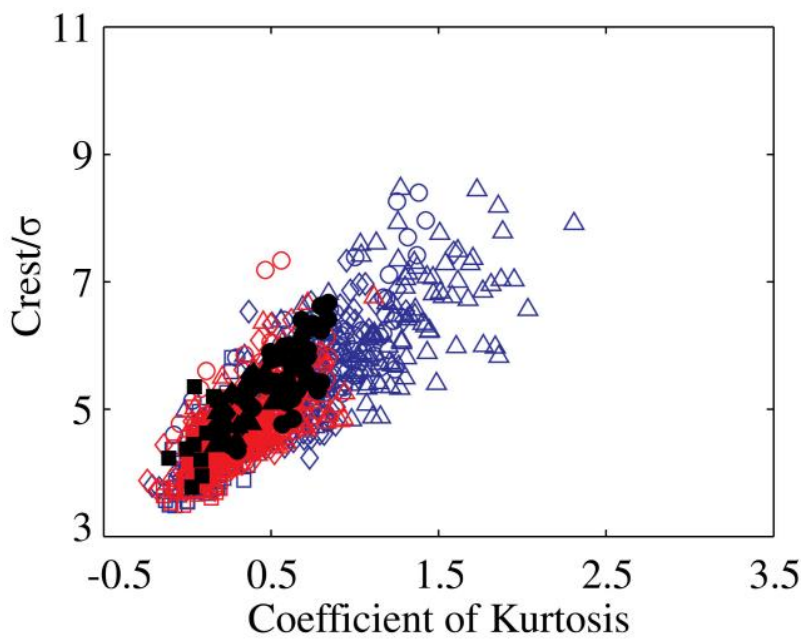

Fig. 5. Relationship between scaled maximal wave crest and coefficient of kurtosis. The meanings of symbols are the same as those in Fig. 3. 

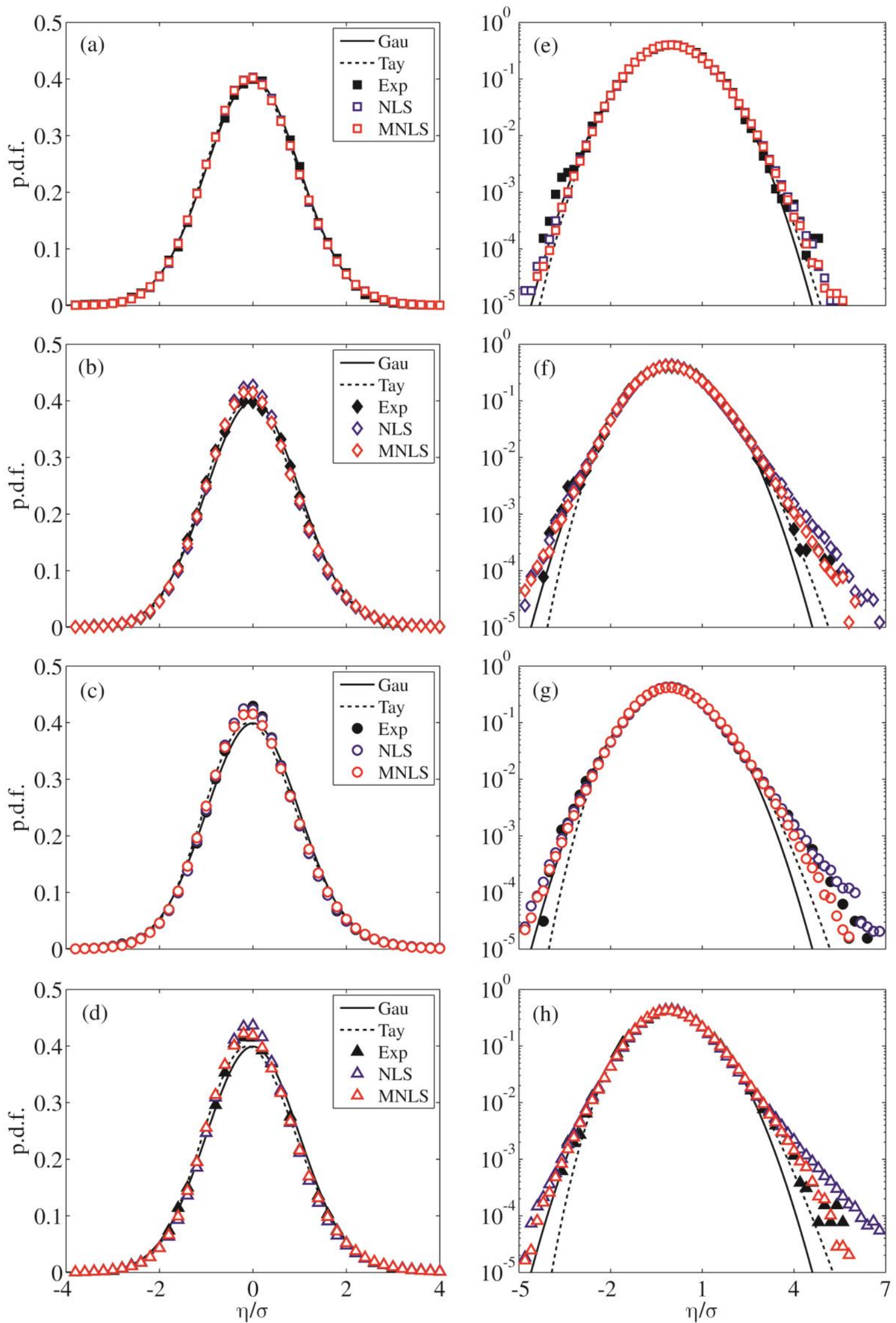

Fig. 6. (a-d) Probability density function of the surface elevations in different sea states in the same location of 
Gauge 8. (e-h) are the same distribution with logarithmic axes.
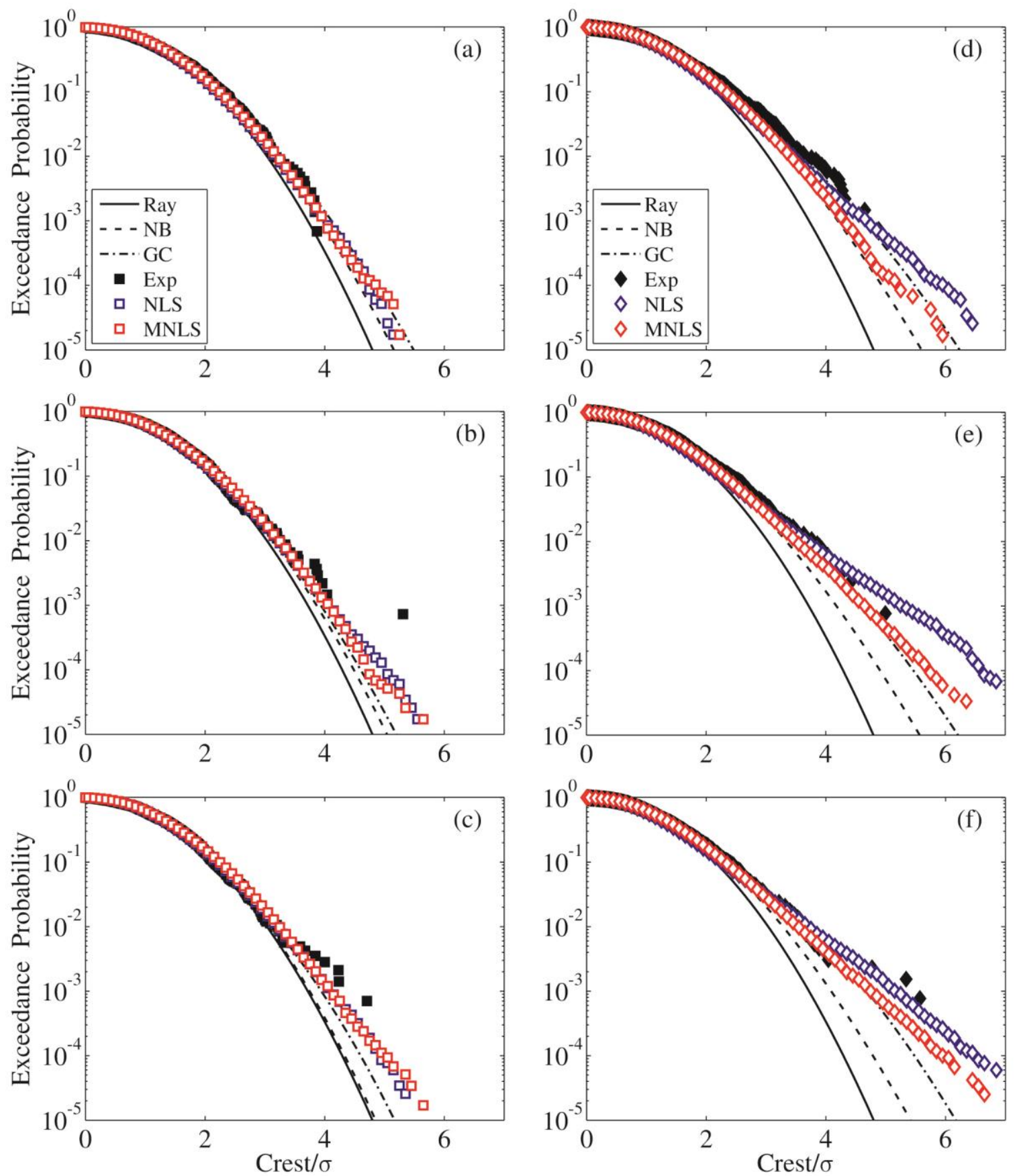

Fig. 7. Exceedance distribution of scaled wave crests in the low (8201) and first moderate (8202) sea states.

(a) (c), (d) (f) correspond to the first two cases listed in Table 1. The three rows present the results obtained from Gauges 1, 5 and 8, respectively. 'Ray', 'NB' and 'GC' represent linear, second-order and third-order models respectively. 

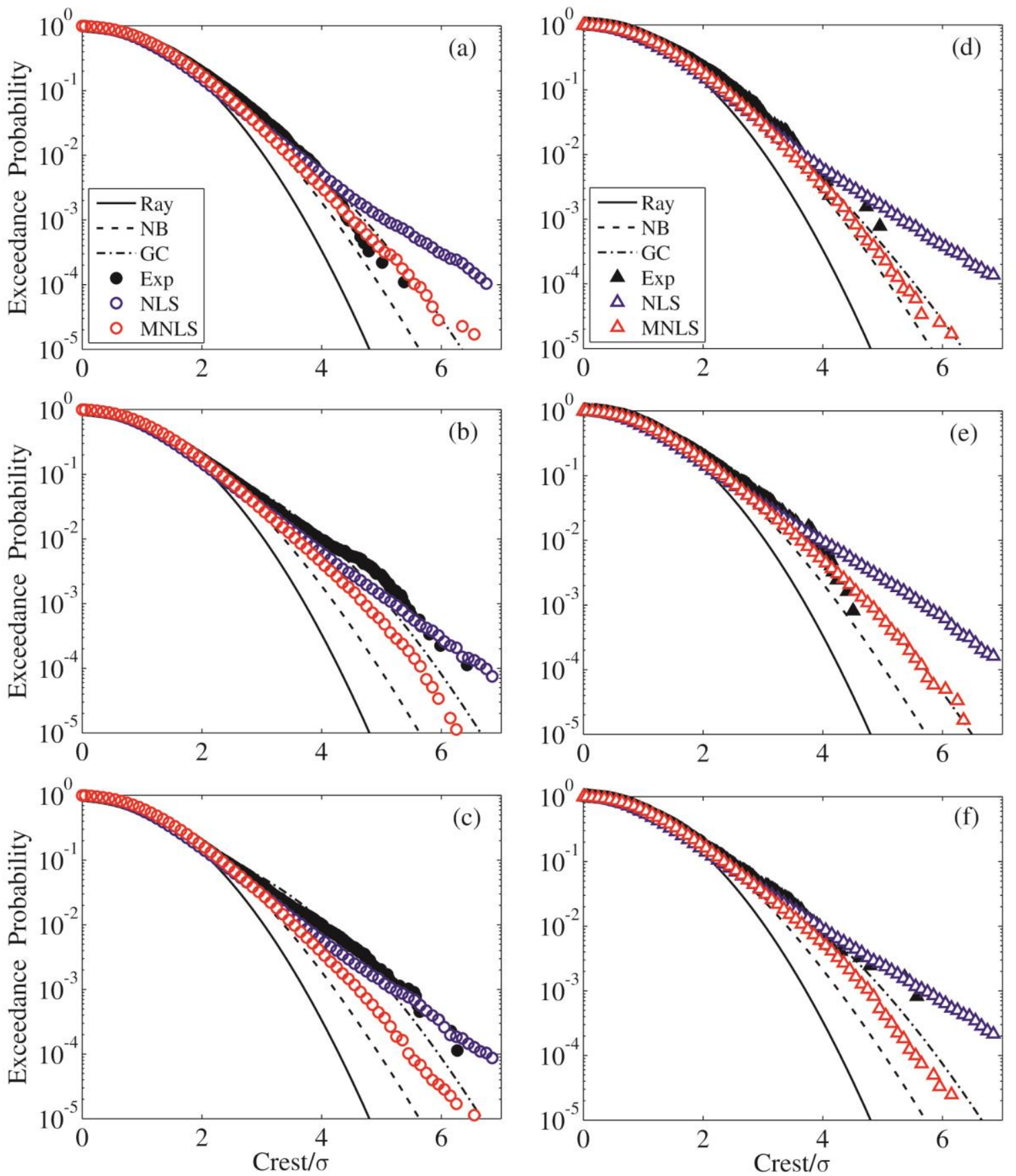

Fig. 8. Exceedance distribution of scaled wave crests in the second moderate (8241) and severe (8219) sea states.

(a) (c), (d) (f) correspond to the last two cases listed in Table 1. The three rows present the results obtained from Gauges 1, 5 and 8, respectively. 'Ray', 'NB' and 'GC' represent linear, second-order and third-order models respectively. 

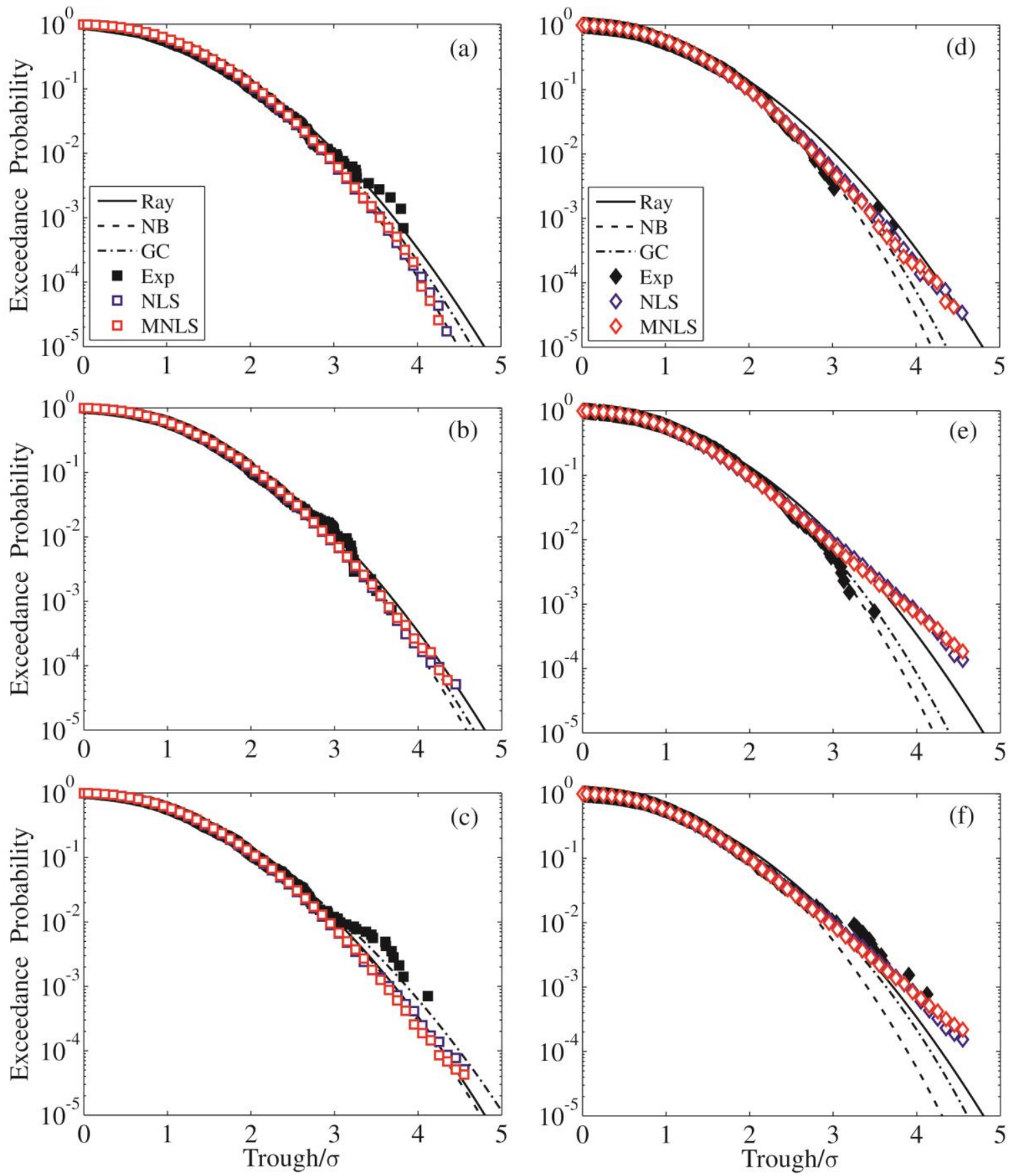

Fig. 9. Exceedance distribution of scaled wave troughs in the low (8201) and first moderate (8202) sea states.

(a) (c), (d) (f) correspond to the first two cases listed in Table 1. The three rows present the results obtained from Gauges 1, 5 and 8, respectively. 'Ray', 'NB' and 'GC' represent linear, second-order and third-order models respectively. 

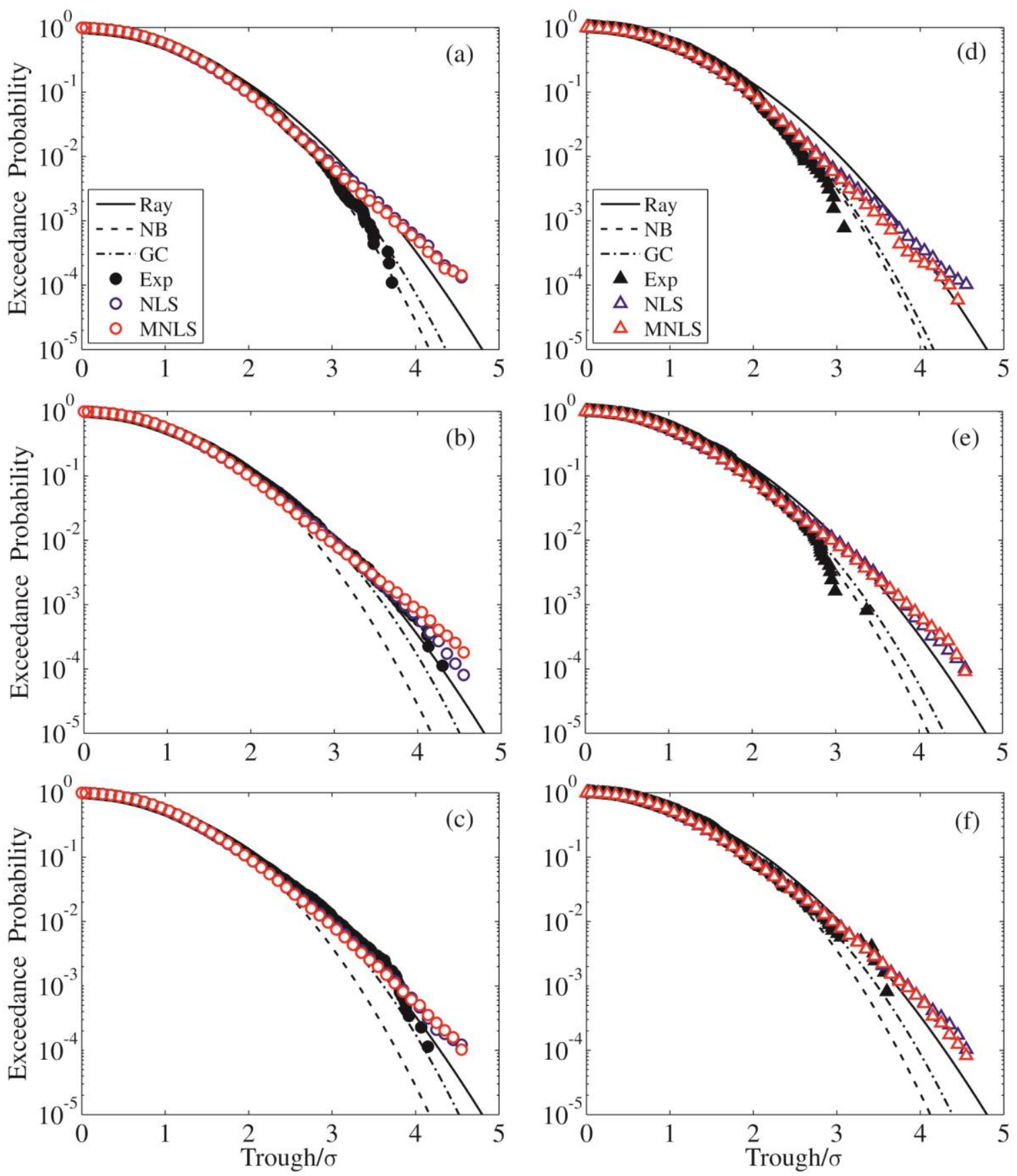

Fig. 10. Exceedance distribution of scaled wave troughs in the second moderate (8241) and severe (8219) sea states. (a) (c), (d) (f) correspond to the last two cases listed in Table 1. The three rows present the results obtained from Gauges 1, 5 and 8, respectively. 'Ray', 'NB' and 'GC' represent linear, second-order and thirdorder models respectively. 

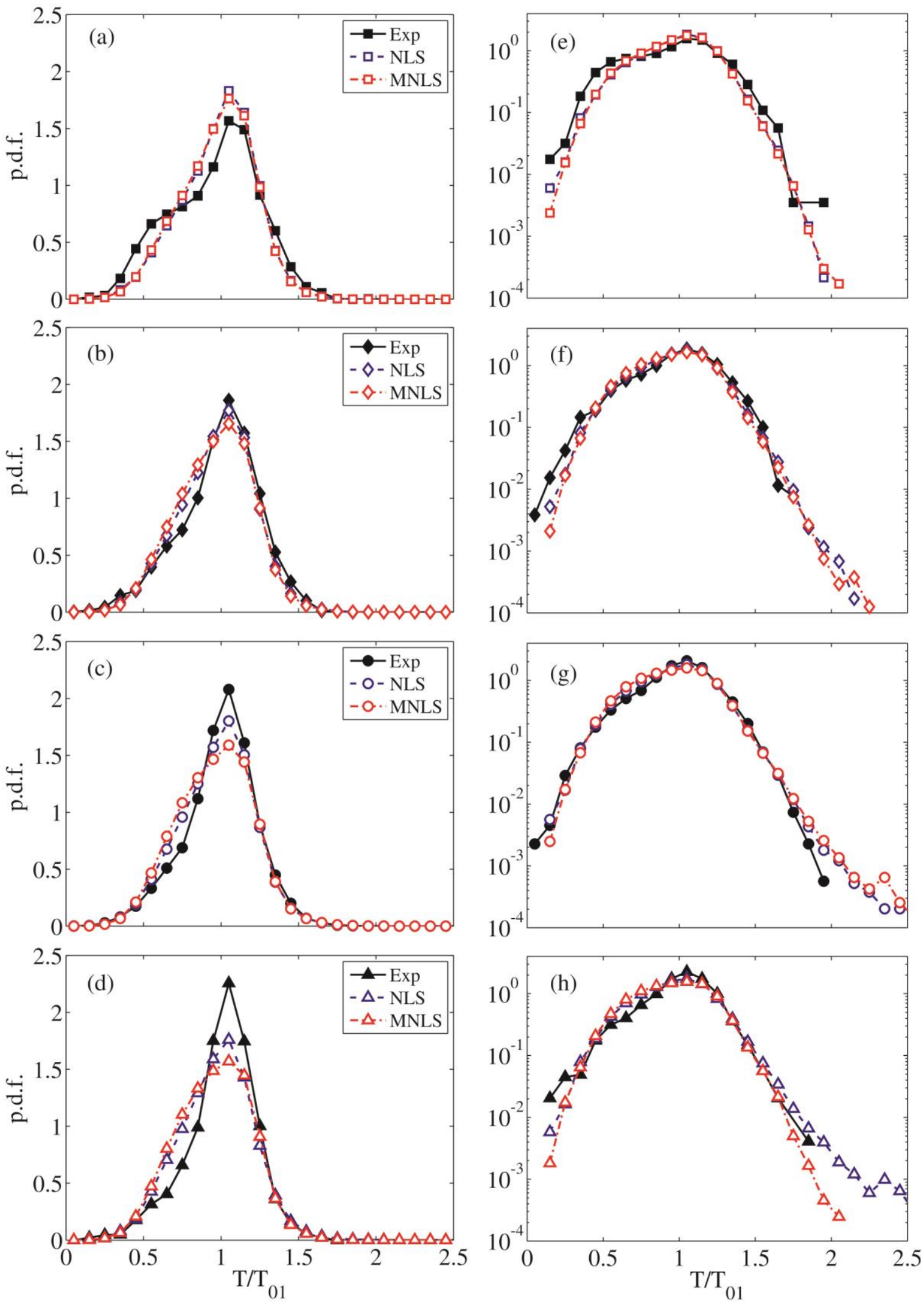

Fig. 11. (a-d) Probability density function of the wave period in different sea states in the same location of Gauge 8. (e-h) are the same distribution with logarithmic axes. 